\title{
ASYMPTOTICALLY CONFORMAL FIXED POINTS AND HOLOMORPHIC MOTIONS
}

\author{
YUNPING JIANG
}

\begin{abstract}
The term integrable asymptotically conformal at a point for a quasiconformal map defined on a domain is defined. Furthermore, we prove that there is a normal form for this kind attracting or repelling or superattracting fixed point with the control condition under a quasiconformal change of coordinate which is also asymptotically conformal at this fixed point. The change of coordinate is essentially unique. These results generalize König's Theorem and Böttcher's Theorem in classical complex analysis. The idea in proofs is new and uses holomorphic motion theory and provides a new understanding of the inside mechanism of these two famous theorems too.
\end{abstract}

\section{INTRODUCTION}

Two of the fundamental theorems in complex dynamical systems are König's Theorem and Bötthcher's Theorem in classical complex analysis which were proved back to 1884 [22] and 1904 [8], respectively, by using some well-known methods in complex analysis. These theorems say that an attracting or repelling or superattracting fixed point of an analytic map can be written into a normal form under a suitable conformal change of coordinate. These theorems become two fundamental results in the recent study of the dynamics of a polynomial or a rational map.

However, it becomes more and more clear in recent years that only conformal changes of coordinate are not enough in the study of many problems in dynamics and in geometry, for examples, in the study of monotonicity of the entropy function for the family $|x|^{3}+t[33$, in the study of deep points and differentiability in hyperbolic 3-manifolds [32, pp.32-34] (see also the end of §3), and in the study of quasiconformal structures on a 4-manifold [11]-in these studies, quasiconformal changes of coordinate are appealed. The quasiconformal changes of coordinate may still have asymptotical conformality property just at one point but definitely not conformal. (It is a big difference between asymptotically conformal and conformal, see definition in Section 2.)

During the study of complex dynamical systems, a subject called holomorphic motions becomes more and more interesting and useful. The subject of holomorphic motions over the open unit disk shows some interesting connections between classical complex analysis and problems on moduli. This subject even becomes an interesting branch in complex analysis [4, 6, 15, 30, 35, 39.

2000 Mathematics Subject Classification. Primary 37F99, Secondary 32H02.

Key words and phrases. integrable asymptotically conformal fixed point, holomorphic motion, normal form.

The research is partially supported by NSF grants and PSC-CUNY awards and the Hundred Talents Program from Academia Sinica. 
In this paper, we will use holomorphic motions over the open unit disk to study the quasiconformal changes of coordinate which are aymptotically conformal at one point. The paper is organized as follows. In Section 2, we give an overview about holomorphic motions and quasiconformal mapping theory. In Section 3, we define an asymptotically conformal fixed point. Moreover, we define an integrable asymptotically conformal fixed point. We then define an attracting or repelling integrable asymptotically conformal fixed point and the control condition. In Section 4, we prove one of our main theorems in this paper:

Theorem 1. Let $f$ be a quasiconformal homeomorphism defined on a neighborhood about 0 . Suppose 0 is an attracting or repelling integrable asymptotically conformal fixed point of $f$ with the control condition. Then there is a quasiconformal homeomorphism $\phi: \Delta_{\delta} \rightarrow \phi\left(\Delta_{\delta}\right) \subset U$ from an open disk of radius $\delta>0$ centered at 0 into $U$ which is asymptotically conformal at 0 such that

$$
\phi^{-1} \circ f \circ \phi(z)=\lambda z, \quad z \in \Delta_{\delta} .
$$

The conjugacy $\phi^{-1}$ is unique up to multiplication of a constant.

To present our idea clearly, we first use the same idea in the proof of above theorem to give a new proof of König's Theorem in classical complex analysis in Section 3. Then we prove Theorem 1 in the same section.

We define an asymptotically conformal super-attracting fixed point in Section 3. In Section 5, we prove the other main theorem in this paper:

Theorem 2. Let $g=f\left(z^{n}\right)$ be a quasiregular map defined on a neighborhood about 0 for $n \geq 2$. Suppose 0 is a super-attracting integrable asymptotically conformal fixed point of $g$. Then there is a quasiconformal homeomorphism $\phi: \Delta_{\delta} \rightarrow \phi\left(\Delta_{\delta}\right) \subset$ $U$ from an open disk of radius $\delta>0$ centered at 0 into $U$ which is asymptotically conformal at 0 such that

$$
\phi^{-1} \circ g \circ \phi(z)=z^{n}, \quad z \in \Delta_{\delta} .
$$

The conjugacy $\phi^{-1}$ is unique up to multiplication by $(n-1)^{\text {th }}$-roots of the unit.

Again, we will first give a new proof of Böttecher's Theorem in classical complex analysis in Section 5. Then we prove Theorem 2 in the same section.

Our proofs in this paper use the "holomorphic motion technique", which we first used in 20. Another place we used the "holomorphic motion technique" is in the study of the Fatou linearization and the quasiconformal rigidity for parabolic germs in 21.

Acknowledgment. I would like to thank Professor Weiyuan Qiu and my students Zhe Wang and Haifeng Chu to help me to clarify several arguments and to fix mistakes and typos in this paper. I would like also to thank Professor Sudeb Mitra who explained to me several points in the development of the measurable Riemann mapping theorem and holomorphic motions.

\section{Holomorphic Motions and Quasiconformal Maps}

In the study of complex analysis, the measurable Riemann mapping theorem plays an important role. Consider the Riemann sphere $\hat{\mathbb{C}}$. A measurable function 
$\mu$ on $\hat{\mathbb{C}}$ is called a Beltrami coefficient if there is a constant $0 \leq k<1$ such that $\|\mu\|_{\infty} \leq k$, where $\|\cdot\|_{\infty}$ means the $L^{\infty}$-norm of $\mu$ on $\hat{\mathbb{C}}$. The equation

$$
H_{\bar{z}}=\mu H_{z}
$$

is called the Beltrami equation with the given Beltrami coefficient $\mu$. The measurable Riemann mapping theorem says that the Beltrami equation has a solution $H$ which is a quasiconformal homeomorphism of $\hat{\mathbb{C}}$ whose quasiconformal dilatation is less than or equal to $K=(1+k) /(1-k)$. The study of the measurable Riemann mapping theorem has a long history since Gauss considered in 1820's the connection with the problem of finding isothermal coordinates for a given surface. As early as 1938, Morrey [36] systematically studied homeomorphic $L^{2}$-solutions of the Beltrami equation (see [27, 28]). But it took almost twenty years until in 1957 Bers [5] observed that these solutions are quasiconformal (refer to [23, pp. $24]$ ). Finally the existence of a solution to the Beltrami equation under the most general possible circumstance, namely, for measurable $\mu$ with $\|\mu\|_{\infty}<1$, was shown by Bojarski [7. In this generality the existence theorem is sometimes called the measurable Riemann mapping theorem (refer to [18, pp. 10].

If one only considers a normalized solution in the Beltrami equation (a solution fixes 0,1 , and $\infty$ ), then $H$ is unique, which is denoted as $H^{\mu}$. The solution $H^{\mu}$ is expressed as a power series made up of compositions of singular integral operators applied to the Beltrami equation on the Riemann sphere. In this expression, if one considers $\mu$ as a variable, then the solution $H^{\mu}$ depends on $\mu$ analytically. This analytic dependence was emphasized by Ahlfors and Bers in their 1960 paper [2] and is essential in determining a complex structure for Teichmüller space (refer to [1, 18, 23, 29, 37]). Note that when $\mu \equiv 0, H^{0}$ is the identity map. A 1-quasiconformal map is conformal. Twenty years later, due to the development of complex dynamics, this analytic dependence presents an even more interesting phenomenon called holomorphic motions as follows.

Let $\Delta_{r}=\{c \in \mathbb{C}|| c \mid<r\}$ be the disk centered at 0 and of radius $r>0$. In particular, we use $\Delta$ to denote the unit disk. Given a Beltrami coefficient $\mu \not \equiv 0$, consider a family of Beltrami coefficients $c \mu /\|\mu\|_{\infty}$ for $c \in \Delta$ and the family of normalized solutions $H \frac{c \mu}{\pi \mu \|_{\infty}}$. Note that $H \frac{c \mu}{\|\mu\|_{\infty}}$ is a quasiconformal homeomorphism whose quasiconformal dilatation is less than or equal to $(1+|c|) /(1-|c|)$. Moreover, $H{ }^{\frac{c \mu}{\mu \mu}}$ is a family which is holomorphic on $c$. Consider a subset $E$ of $\hat{\mathbb{C}}$ and its image $E_{c}=H \frac{c \mu}{\|\mu\| \infty}(E)$. One can see that $E_{c}$ moves holomorphically in $\hat{\mathbb{C}}$ when $c$ moves in $\Delta$. That is, for any point $z \in E, z(c)=H{ }^{\frac{c \mu}{\| \mu}}(z)$ traces a holomorphic path starting from $z$ as $c$ moves in the unit disk. Although $E$ may start out as smooth as a circle and although the points of $E$ move holomorphically, $E_{c}$ can be an interesting fractal with fractional Hausdorff dimension for every $c \neq 0$ (see [17]).

Surprisingly, the converse of the above fact is true too. This starts from the famous $\lambda$-lemma of Mañé, Sad, and Sullivan [31] in complex dynamical systems. Let us start to understand this fact by first defining holomorphic motions.

Definition 1 (Holomorphic Motions). Let $E$ be a subset of $\hat{\mathbb{C}}$. Let

$$
h(c, z): \Delta_{r} \times E \rightarrow \hat{\mathbb{C}}
$$

be a map. Then $h$ is called a holomorphic motion of $E$ parametrized by $\Delta_{r}$ if

(1) $h(0, z)=z$ for $z \in E$;

(2) for any fixed $c \in \Delta_{r}, h(c, \cdot): E \rightarrow \hat{\mathbb{C}}$ is injective; 
(3) for any fixed $z, h(\cdot, z): \Delta_{r} \rightarrow \hat{\mathbb{C}}$ is holomorphic.

For example, for a given Beltrami coefficient $\mu$,

$$
H(c, z)=H^{\frac{c \mu}{\|\mu\|_{\infty}}}(z): \Delta \times \hat{\mathbb{C}} \rightarrow \hat{\mathbb{C}}
$$

is a holomorphic motion of $\hat{\mathbb{C}}$ parametrized by $\Delta$.

Note that even continuity does not directly enter into the definition; the only restriction is in the $c$ direction. However, continuity is a consequence of the hypotheses from the proof of the $\lambda$-lemma of Mañé, Sad, and Sullivan [31, Theorem 2]. Moreover, Mañé, Sad, and Sullivan prove in [31] that

Lemma 1 ( $\lambda$-Lemma). A holomorphic motion $h(c, z): \Delta \times E \rightarrow \hat{\mathbb{C}}$ of a set $E \subset \hat{\mathbb{C}}$ parametrized by $\Delta$ can be extended to a holomorphic motion $H(c, z): \Delta \times \bar{E} \rightarrow \hat{\mathbb{C}}$ of the closure $\bar{E}$ of $E$ parametrized by the same $\Delta$.

Furthermore, Mañé, Sad, and Sullivan show in 31] that $H(c, \cdot): \bar{E} \rightarrow \hat{\mathbb{C}}$ satisfies the Pesin property. In particular, when $\bar{E}$ is a closed domain, this property can be described as the quasiconformal property. A further study of this quasiconformal property is given by Sullivan and Thurston [39] and Bers and Royden [6]. In [39], Sullivan and Thurston prove that there is a universal constant $a>0$ such that any holomorphic motion of any set $E \subset \hat{\mathbb{C}}$ parametrized by the open unit disk $\Delta$ can be extended to a holomorphic motion of $\hat{\mathbb{C}}$ parametrized by $\Delta_{a}$. In [6], Bers and Royden show, by using classical Teichmüller theory, that this constant actually can be taken to be $1 / 3$. Moreover, in the same paper, Bers and Royden show that in any holomorphic motion $H(c, z): \Delta \times \hat{\mathbb{C}} \rightarrow \hat{\mathbb{C}}, H(c, \cdot): \hat{\mathbb{C}} \rightarrow \hat{\mathbb{C}}$ is a quasiconformal homeomorphism whose quasiconformal dilatation less than or equal to $(1+|c|) /(1-|c|)$ for $c \in \Delta$. In the both papers [39, 6], they expect $a=1$. This was eventually proved by Slodkowski in [38].

Theorem 3 (The Holomorphic Motion Theorem). Suppose $h(c, z): \Delta \times E \rightarrow \hat{\mathbb{C}}$ is a holomorphic motion of a closed subset $E$ of $\hat{\mathbb{C}}$ parameterized by the unit disk $\Delta$. Then there is a holomorphic motion $H(c, z): \Delta \times \hat{\mathbb{C}} \rightarrow \hat{\mathbb{C}}$ which extends $h(c, z): \Delta \times E \rightarrow \hat{\mathbb{C}}$. Moreover, for any fixed $c \in \Delta, H(c, \cdot): \hat{\mathbb{C}} \rightarrow \hat{\mathbb{C}}$ is a quasiconformal homeomorphism whose quasiconformal dilatation

$$
K(H(c, \cdot)) \leq \frac{1+|c|}{1-|c|} .
$$

The Beltrami coefficient of $H(c, \cdot)$ given by

$$
\mu(c, z)=\frac{\partial H(c, z)}{\partial \bar{z}} / \frac{\partial H(c, z)}{\partial z}
$$

is a holomorphic function from $\Delta$ into the unit ball of the Banach space $\mathcal{L}^{\infty}(\mathbb{C})$ of all essentially bounded measurable functions on $\mathbb{C}$.

Chirka gives a nice proof of Slodkowski's theorem by using some results in functional analysis. The reader can find a complete proof of the above holomorphic motion theorem in [16] following the ideas in Chirka's proof [10] and in Bers-Royden's proof [6]. Moreover, some property of infinitesimal holomorphic motions is discussed in [16]. 
Holomorphic motions of a set $E \subset \hat{\mathbb{C}}$ parametrized by a connected complex manifold with a base point can be also defined. They have many interesting relationships with the Teichmüller space $T(E)$ of a closed set $E$ of the Riemann sphere $\hat{\mathbb{C}}$ (refer to [35]).

In addition to the references we mentioned above, there is a partial list of references [3, 4, 12, 13, 14, 15, 30, 24, 25, about holomorphic motions and Teichmüller theory. The reader who is interested in holomorphic motions may refer to those papers and books.

\section{INTEGRABLE ASYMPTOTICALLY CONFORMAL FIXED POINTS}

Let $f$ be a quasiconformal homeomorphism defined on a domain $U$ in the Riemann sphere $\hat{\mathbb{C}}$. Suppose $p$ is a point in the $U$. Let $\Delta_{t}(p)$ denote the disk of radius $t>0$ centered at $p$. Let $\mu_{f}(z)=f_{\bar{z}} / f_{z}$ be the complex dilatation of $f$ on $U$. Suppose $t_{0}>0$ be a number such that $\Delta_{t_{0}}(p) \subset U$. Then for any $0<t \leq t_{0}$, let $\omega_{f, p}(t)=\left\|\mu_{f} \mid \Delta_{t}(p)\right\|_{\infty}$, where $\|\cdot\|_{\infty}$ means the $L^{\infty}$ norm.

Definition 2. We call $f$ asymptotically conformal at $p$ if

$$
\omega_{f, p}(t) \rightarrow 0 \text { as } t \rightarrow 0^{+} .
$$

Furthermore, we call $f$ integrable asymptotically conformal at $p$ if

$$
\int_{0}^{t_{0}} \frac{\omega_{f, p}(s)}{s} d s<\infty
$$

If $f$ is asymptotically conformal at $p$, then $f$ maps a tiny circle centered at $p$ to an ellipse centered at $f(p)$ and, moreover, the ratio of the long axis and the short axis tends to 1 as the radius of the tiny circle tends to 0 . But the map still can fail to be differentiable at $p$ (refer to [19]). However, following Reshetnyak's 1978 paper [26. Theorem 1.1, pp. 204]), if $f$ is integrable asymptotically conformal at $p$, then $f$ is differentiable and conformal at $p$, i.e., the limit of $(f(z)-f(p)) /(z-p)$ exists as $z$ goes to $p$. If, in addition, $p$ is a fixed point of $f$, that is, $f(p)=p$, let

$$
\lambda=\lim _{z \rightarrow p} \frac{f(z)-f(p)}{z-p}
$$

and call it the multiplier of $f$ at $p$. We call $p$

i) attracting if $0<|\lambda|<1$;

ii) repelling if $|\lambda|>1$;

iii) neutral if $|\lambda|=1$.

Correspondingly, we call $p$ an attracting, repelling, or neutral integrable asymptotically conformal fixed point of $f$. By linear changes of coordinate, we can assume that $p=f(p)=0$. We will keep this assumption without loss of generality.

Let $g$ be a quasiregular map defined on a neighborhood $U$ of 0 fixing 0 . Assume $g=f \circ q_{n}$ where $q_{n}(z)=z^{n}, n \geq 2$, and $f$ is a quasiconformal homeomorphism. We say $g$ is integrable asymptotically conformal at 0 if $f$ is integrable asymptotically conformal at 0 with nonzero multiplier

$$
\lambda=\lim _{z \rightarrow 0} \frac{f(z)}{z} .
$$

In this case, 0 is called a super-attracting integrable asymptotically conformal fixed point of $g$. 
The following lemma will be useful in our proofs of Theorems 1 and 2 .

Lemma 2. Suppose $\omega(t)$ is an increasing function of $0<t \leq t_{0}$. Suppose

$$
\int_{0}^{t_{0}} \frac{\omega(s)}{s} d s<\infty
$$

Suppose $0<\sigma<1$ and $C>0$ are two constants. Let

$$
\tilde{\omega}(t)=\sum_{n=0}^{\infty} \omega\left(C \sigma^{n} t\right)
$$

for all $t>0$ such that $C t \leq t_{0}$. Then

$$
\tilde{\omega}(t) \leq \omega(C t)+\frac{1}{-\log \sigma} \int_{0}^{C t} \frac{\omega(s)}{s} d s .
$$

Moreover, $\tilde{\omega}(t) \rightarrow 0$ as $t \rightarrow 0^{+}$.

Proof. Since $\omega(t)$ is increasing for $t>0$, we have

$\tilde{\omega}(t)=\omega(C t)+\sum_{n=1}^{\infty} \omega\left(C \sigma^{n} t\right) \leq \omega(C t)+\sum_{n=1}^{\infty} \int_{n-1}^{n} \omega\left(C \sigma^{x} t\right) d x=\omega(C t)+\int_{0}^{\infty} \omega\left(C \sigma^{x} t\right) d x$.

Let $s=C \sigma^{x} t$. Then $d s=(\log \sigma) s d x$. We have that

$$
\int_{0}^{\infty} \omega\left(C \sigma^{x} t\right) d x=\frac{1}{-\log \sigma} \int_{0}^{C t} \frac{\omega(s)}{s} d s
$$

It is interesting to compare our integrable asympotically conformal at a point to

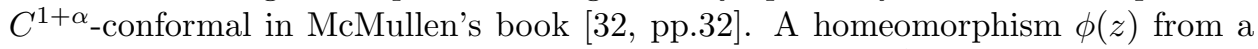
neighborhood $U$ of $\mathbb{C}$ to another neighborhood $V$ of $\mathbb{C}$ is $C^{1+\alpha}$-conformal at $p \in U$ for some $0<\alpha \leq 1$ if the complex derivative $\phi^{\prime}(p)$ exists and

$$
\phi(z+p)=\phi(p)+\phi^{\prime}(p) z+O\left(|z|^{1+\alpha}\right)
$$

for all $z \in \mathbb{C}$ sufficiently small.

For a given Kleinian group $\Gamma$ preserving the upper-half space $\mathbb{H}^{3}$ such that the 3-manifold $M^{3}=\mathbb{H}^{3} / \Gamma$ has the bounded geometry, that is, its injectivity radius is bounded above and below in its convex core. It is proven that any quasiconformal conjugacy from $\Gamma$ to another Kleinian group $\Gamma^{\prime}$ is $C^{1+\alpha}$-conformal at every deep point in the limit set $\Lambda$ of $\Gamma$ (see [32, Theorem 2.18]). This theorem can be thought of as an extension of Mostow rigidity at every deep point when the limit set is not the whole sphere.

Suppose $\phi: U \rightarrow V$ is quasiconformal and $C^{1+\alpha}$-conformal at $p$ for some $0<$ $\alpha \leq 1$. By linear changes of coordinate, we can assume $p=0$ and $\phi(p)=0$. Then

$$
\phi(z)=\phi^{\prime}(0) z+O\left(|z|^{1+\alpha}\right)
$$

for $z \in \mathbb{C}$ sufficiently small. Since a quasiconformal homeomorphism is differentiable almost everywhere, $F(z)=O\left(|z|^{1+\alpha}\right)$ is differentiable almost everywhere. Suppose $F(z)=H\left(|z|^{1+\alpha}\right)$. Then $H^{\prime}(x)$ exists for almost every $x$. Suppose $H^{\prime}(x)$ is a bounded function. At every differentiable point $z \neq 0$, we have that

$$
\left|\frac{\partial \phi(z)}{\partial \bar{z}}\right|=\left|\frac{\partial F(z)}{\partial \bar{z}}\right| \leq C\left|z^{\frac{\alpha+1}{2}}(\bar{z})^{\frac{\alpha-1}{2}}\right|
$$


and

$$
\left|\frac{\partial \phi(z)}{\partial z}\right|=\left|\phi^{\prime}(0)+\frac{\partial F(z)}{\partial z}\right| \geq\left|\phi^{\prime}(0)\right|-C\left|z^{\frac{\alpha-1}{2}}(\bar{z})^{\frac{\alpha+1}{2}}\right|
$$

where $C>0$ is a constant. Since $\phi^{\prime}(0) \neq 0$, we get that for $z \in \mathbb{C}$ sufficiently small,

$$
\left|\mu_{\phi}(z)\right|=\left|\frac{\partial \phi(z)}{\partial \bar{z}} / \frac{\partial \phi(z)}{\partial z}\right| \leq C^{\prime}|z|^{\alpha}
$$

where $C^{\prime}>0$ is another constant. Then

$$
\omega_{\phi, 0}(t)=\left\|\mu_{\phi} \mid \Delta_{t}\right\|_{\infty} \leq C^{\prime} t^{\alpha} .
$$

Thus $\phi$ is integrable asymptotically conformal at 0 .

If $\phi$ is quasiconformal in a neighborhood $U$ of 0 fixing 0 and $C^{1+\alpha}$-conformal at 0 for some $0<\alpha \leq 1$, then it will automatically satisfy the control condition (11) in the next section as follows.

Suppose $\lambda=\phi^{\prime}(0)$ and suppose $0<|\lambda|<1$. (If $|\lambda|>1$, then we consider $\phi^{-1}$.) Choose a constant $0<a<1$ such that $a^{1+\alpha}<|\lambda|<a$. We can choose a $\delta>0$ such

that $\bar{\Delta}_{\delta} \subset U$ and such that $|\phi(z)| \leq a|z|$ for any $z \in \bar{\Delta}_{\delta}$. Then there is a constant $C_{0}>0$ such that, for any $|z| \leq \delta$,

$$
\left|\phi^{n+1}(z)-\lambda \phi^{n}(z)\right| \leq C_{0}\left|\phi^{n}(z)\right|^{1+\alpha} \leq C_{0}|z|^{1+\alpha} a^{n(1+\alpha)} .
$$

Let $0<\tau=a^{1+\alpha} /|\lambda|<1$ and $C_{1}=C_{0} \delta^{\alpha} /|\lambda|$. Then

$$
\left|\frac{\phi^{n+1}(z)}{\lambda^{n+1} z}-\frac{\phi^{n}(z)}{\lambda^{n} z}\right| \leq C_{1} \tau^{n}
$$

Thus $\left\{h_{n}(z)=\phi^{n}(z) /\left(\lambda^{n} z\right)\right\}_{n=0}^{\infty}$ is a uniform Cauchy sequence of continuous functions defined on $\bar{\Delta}_{\delta}$. Furthermore, $h_{n}(0)=1$ for all $n \geq 0$. Thus there is a constant $C>0$ such that

$$
C^{-1} \leq\left|h_{n}(z)\right|=\left|\frac{\phi^{n}(z)}{\lambda^{n} z}\right| \leq C
$$

for all $z \in \bar{\Delta}_{\delta}$ and all $n \geq 0$ as long as $\delta$ small enough. Therefore, if $\phi(z)$ is quasiconformal and $C^{1+\alpha}$-conformal at 0 , then it satisfies all assumptions in Theorem 1

\section{LiNEARIZATION FOR INTEGRABLE ASYMPTOTICALLY CONFORMAL ATTRACTING OR REPELLING FIXED POINTS}

One of the main results in this article is Theorem 1, which says that if $f$ is a quasiconformal homeomorphism on a neighborhood $U$ of 0 and 0 is an attracting or repelling integrable asymptotically conformal fixed point with the multiplier $\lambda$, $0<|\lambda|<1$ or $|\lambda|>1$ and with the control condition, then $f$ can be written as a linear map $z \rightarrow \lambda z$ under some quasiconformal change of coordinate which is also asymptotically conformal at 0 . We only need to consider the attracting case because that in the repelling case, we can consider $f^{-1}$. In the attracting case, we say $f$ satisfies the control condition if there are constants $\delta>0$ and $C>0$ such that

$$
C^{-1} \leq\left|\frac{f^{n}(z)}{\lambda^{n} z}\right| \leq C
$$

for all $z \in \bar{\Delta}_{\delta} \subset U$ and all $n \geq 0$.

The result generalizes the famous König's Theorem in classical analysis. Therefore, to present a clear idea about how we get Theorem 1, we first use the same idea to give another proof of König's Theorem, which is first given in [20. The idea 
of the new proof follows the viewpoint of holomorphic motions. For the classical proof of König's Theorem, the reader may refer to König's original paper [22] or most recent books [9, 34. Actually from the technical point of views, our proof is more complicate and uses a sophistical result. But from the conceptual point of views, our proof gives some inside mechanism for the linearization of an attracting or a repelling fixed point.

Theorem 4 (König's Theorem). Let $f(z)=\lambda z+\sum_{j=2}^{\infty} a_{j} z^{j}$ be an analytic function defined on $\Delta_{r_{0}}, r_{0}>0$. Suppose $0<|\lambda|<1$ or $|\lambda|>1$. Then there is a conformal map $\phi: \Delta_{\delta} \rightarrow \phi\left(\Delta_{\delta}\right)$ for some $0<\delta<r_{0}$ such that

$$
\phi^{-1} \circ f \circ \phi(z)=\lambda z \text {. }
$$

The conjugacy $\phi^{-1}$ is unique up to multiplication of a constant.

A new proof of Theorem 4. We only need to prove it for $0<|\lambda|<1$. In the case of $|\lambda|>1$, we can consider $f^{-1}$.

First, we can find a $0<\delta<r_{0}$ such that

$$
|f(z)|<|z|, \quad z \in \bar{\Delta}_{\delta}
$$

and $f$ is injective on $\bar{\Delta}_{\delta}$. For every $0<r \leq \delta$, let

$$
S_{r}=\{z \in \mathbb{C}|| z \mid=r\}
$$

and

$$
T_{r}=|\lambda| S_{r}=\{z \in \mathbb{C}|| z|=| \lambda \mid r\} .
$$

Denote $E=S_{r} \cup T_{r}$. Define

It is clear that

$$
\phi_{r}(z)= \begin{cases}z & z \in S_{r} \\ f\left(\frac{z}{\lambda}\right), & z \in T_{r}\end{cases}
$$

$$
\phi_{r}^{-1} \circ f \circ \phi_{r}(z)=\lambda z
$$

for $z \in S_{r}$.

Now write $\phi_{r}(z)=z \psi_{r}(z)$ for $z \in T_{r}$, where

$$
\psi(z)=1+\sum_{j=1}^{\infty} \frac{a_{j+1}}{\lambda^{j+1}} z^{j} .
$$

Define

Note that

$$
h_{r}(c, z)=\left\{\begin{array}{ll}
z, & z \in S_{r} \\
z \psi\left(\frac{\delta c z}{r}\right), & z \in T_{r}
\end{array}: \Delta \times E \rightarrow \hat{\mathbb{C}} .\right.
$$

$$
h_{r}(c, z)=z \psi\left(\frac{c z \delta}{r}\right)=\frac{r}{c \delta} f\left(\frac{c z \delta}{r \lambda}\right), \quad z \in T_{r}, \quad c \neq 0 .
$$

For each fixed $z \in E$, it is clear that $h(c, z)$ is a holomorphic function of $c \in \Delta$. For each fixed $c \in \Delta$, the restriction $h(c, \cdot)$ to $S_{r}$ and $T_{r}$, respectively, are injective. Now we claim that their images do not cross either. That is because for any $z \in T_{r}$, $|z|=|\lambda| r$ and $|c z \delta| /|r \lambda| \leq \delta$, so

$$
|h(c, z)|=\left|\frac{r}{c \delta}\right|\left|f\left(\frac{c z \delta}{r \lambda}\right)\right|<\left|\frac{r}{c \delta}\right|\left|\frac{c z \delta}{r \lambda}\right|=r .
$$

Therefore, $h(c, z): \Delta \times E \rightarrow \hat{\mathbb{C}}$ is a holomorphic motion because we also have $h(0, z)=z$ for all $z \in E$. From Theorem $3, h$ can be extended to a holomorphic 
motion $H(c, z): \Delta \times \hat{\mathbb{C}} \rightarrow \hat{\mathbb{C}}$, and moreover, for each fixed $c \in \Delta, H(c, \cdot): \hat{\mathbb{C}} \rightarrow \hat{\mathbb{C}}$ is a quasiconformal homeomorphism whose quasiconformal dilatation is less than or equal to $(1+|c|) /(1-|c|)$. Now take $c_{r}=r / \delta$ and consider $H\left(c_{r}, \cdot\right)$. We have $H\left(c_{r}, \cdot\right) \mid E=\phi_{r}$. Let

$$
A_{r, j}=\left\{\left.z \in \mathbb{C}|| \lambda\right|^{j+1} r \leq|z| \leq|\lambda|^{j} r\right\} .
$$

We still use $\phi_{r}$ to denote $H\left(c_{r}, \cdot\right) \mid A_{r, 0}$.

For an integer $k>0$, take $r=r_{k}=\delta|\lambda|^{k}$. Then

$$
\bar{\Delta}_{\delta}=\cup_{j=-k}^{\infty} A_{r, j} \cup\{0\} .
$$

Extend $\phi_{r}$ to $\bar{\Delta}_{\delta}$, which we still denote as $\phi_{r}$, as follows.

$$
\phi_{r}(z)=f^{j}\left(\phi_{r}\left(\lambda^{-j} z\right)\right), \quad z \in A_{r, j}, \quad j=-k, \cdots,-1,0,1, \cdots,
$$

and $\phi_{r}(0)=0$. Since $\phi_{r} \mid E$ is a conjugacy from $f$ to $\lambda z, \phi_{r}$ is continuous on $\Delta_{\delta}$. Since $f$ is conformal, $\phi_{r}$ is quasiconformal whose quasiconformal dilatation is the same as that of $H\left(c_{r}, \cdot\right)$ on $A_{r, 0}$. So the quasiconformal dilatation of $\phi_{r}$ on $\Delta_{\delta}$ is less than or equal to $\left(1+c_{r}\right) /\left(1-c_{r}\right)$. Furthermore,

$$
f\left(\phi_{r}(z)\right)=\phi_{r}(\lambda z), \quad z \in \Delta_{\delta} .
$$

Since $f^{-1}(z)=\lambda^{-1} z(1+O(z)), f^{-k}(z)=\lambda^{-k} z \prod_{i=0}^{k-1}\left(1+O\left(\lambda^{-i} z\right)\right)$. Because $|\lambda|^{-k} r_{k}=\delta$, the range of $\phi_{r_{k}}$ on $\Delta_{\delta}$ is a Jordan domain bounded above from $\infty$ and below from 0 uniformly on $k$. In addition, 0 is fixed by $\phi_{r_{k}}$ and the quasiconformal dilatations of the $\phi_{r_{k}}$ are uniformly bounded. Therefore, the sequence $\left\{\phi_{r_{k}}\right\}_{k=1}^{\infty}$ is in a compact set in the space of all quasiconformal homeomorphisms on $\Delta_{\delta}$ (see [1]). Let $\phi$ be a limiting map of this family. Then we have

$$
f(\phi(z))=\phi(\lambda z), \quad z \in \Delta_{\delta}
$$

The quasiconformal dilatation of $\phi$ is less than or equal to $\left(1+c_{r_{k}}\right) /\left(1-c_{r_{k}}\right)$ for all $k>0$. So $\phi$ is a 1-quasiconformal map, and thus is conformal. This is the proof of the existence.

For the sake of completeness, we also provide the proof of uniqueness but this is not new and the reader can find it on [9, 34]. Suppose $\phi_{1}$ and $\phi_{2}$ are two conjugacies such that

$$
\phi_{1}^{-1} \circ f \circ \phi_{1}(z)=\lambda z \quad \text { and } \quad \phi_{2}^{-1} \circ f \circ \phi_{2}(z)=\lambda z, \quad z \in \Delta_{\delta} .
$$

Then for $\Phi=\phi_{2}^{-1} \circ \phi_{1}$, we have $\Phi(\lambda z)=\lambda \Phi(z)$. This implies that $\Phi^{\prime}(\lambda z)=\Phi^{\prime}(z)$ for any $z \in \Delta_{\delta}$. Thus $\Phi^{\prime}(z)=\Phi^{\prime}\left(\lambda^{n} z\right)=\Phi(0)=0$. So $\Phi(z)=$ const and $\phi_{2}^{-1}=$ const. $\cdot \phi_{1}^{-1}$.

Now let us prove Theorem 1 .

Proof of Theorem 1, We need only to prove this theorem for attracting integrable asymptotically conformal germs. In the case of repelling integrable asymptotically conformal germs, we can consider $f^{-1}$.

Let $\sigma=|\lambda|$. First, we can find a $\delta>0$ such that $\bar{\Delta}_{\delta} \subset U, f$ is injective on $\bar{\Delta}_{\delta}$,

$$
|f(z)|<|z|, \quad z \in \bar{\Delta}_{\delta},
$$

and the control condition (10) is held on $\bar{\Delta}_{\delta}$.

For every $0<r \leq \delta$, let

$$
S_{r}=\{z \in \mathbb{C}|| z \mid=r\}
$$


and

$$
T_{r}=\sigma S_{r}=\{z \in \mathbb{C}|| z \mid=\sigma r\} .
$$

Denote $E=S_{r} \cup T_{r}$. Define

$$
\phi_{r}(z)= \begin{cases}z & z \in S_{r} \\ f\left(\frac{z}{\lambda}\right), & z \in T_{r}\end{cases}
$$

It is clear that

$$
\phi_{r}^{-1} \circ f \circ \phi_{r}(z)=\lambda z
$$

for $z \in S_{r}$.

Now write $\phi(z)=f\left(\frac{z}{\lambda}\right)$ defined on $\Delta_{r}$. Suppose $\phi(r)=\tau_{r}$. Extend $\phi$ to $\hat{\mathbb{C}}$ by quasiconformal reflection with respect to $S_{r}$ and $\phi\left(S_{r}\right)$ (see [1]). We still denote this extended map as $\phi$. Let $\nu=\phi_{\bar{z}} / \phi_{z}$ be the complex dilatation of the extended $\phi$. Then

$$
a(r)=\|\nu\|_{\infty}=O\left(\left\|\mu \mid \Delta_{\sigma^{-1} r}\right\|_{\infty}\right)=O\left(\omega\left(\sigma^{-1} r\right)\right) .
$$

Consider $\nu_{c}=c a_{0} a(r)^{-1} \nu$ and the unique solution $\phi_{c}=\phi^{\nu_{c}}$ that maps $0, r$, and $\infty$ to $0, \tau_{r}$, and $\infty$, respectively. Here $a_{0}$ is a constant independent of $r$ such that $\left|\phi_{c}(z)\right|<r$ for all $|z| \leq \sigma r$ and $|c|<1$. (Since $\phi_{c}$ can be written as a power series in $c$ and $\left\|\nu_{c}\right\| \rightarrow 0$ uniformly as $r \rightarrow 0$, such an $a_{0}$ exists.) Then $\phi_{c}$ holomorphically depends on $c \in \Delta$. Define

$$
\phi_{r}(c, z)= \begin{cases}z & z \in S_{r} \\ \phi_{c}(z), & z \in T_{r} .\end{cases}
$$

It is a holomorphic motion from $\Delta \times E \rightarrow \hat{\mathbb{C}}$. From Theorem 3, $\phi_{r}(c, z)$ can be extended to a holomorphic motion from $\Delta \times \hat{\mathbb{C}} \rightarrow \hat{\mathbb{C}}$, which we still denote by $\phi_{r}(c, z)$, such that the quasiconformal dilatation of $\phi_{r}(c, \cdot)$ is less than or equal to $(1+|c|) /(1-|c|)$. In particular when $c_{r}=a_{0}^{-1} a(r), \phi_{r}\left(c_{r}, z\right) \mid E=\phi_{r}$. Let

$$
A_{r, j}=\left\{z \in \mathbb{C}\left|\sigma^{j+1} r \leq\right| z \mid \leq \sigma^{j} r\right\} .
$$

We still use $\phi_{r}$ to denote $\phi_{r}\left(c_{r}, \cdot\right) \mid A_{r, 0}$. For an integer $k>0$, take $r=r_{k}=\delta \sigma^{k}$. Then

$$
\Delta_{\delta}=\cup_{j=-k}^{\infty} A_{r, j} \cup\{0\} .
$$

Extend $\phi_{r}$ to $\Delta_{\delta}$, which we still denote as $\phi_{r}$, by

$$
\phi_{r}(z)=f^{j}\left(\phi_{r}\left(\lambda^{-j} z\right)\right), \quad z \in A_{r, j}, \quad j=-k, \cdots,-1,0,1, \cdots,
$$

and $\phi_{r}(0)=0$. Since $\phi_{r} \mid E$ is a conjugacy from $f$ to $\lambda z, \phi_{r}$ is continuous on $\Delta_{\delta}$.

Next we need to estimate the quasiconformal constant of $\phi_{r}$ on $\Delta_{\delta}$. We will use the following formula (refer to [1]): If $F$ and $G$ are two quasiconformal maps with the complex dilatations $\mu_{F}$ and $\mu_{G}$. Then the composition map $G \circ F$ has the complex dilatation

$$
\mu_{G \circ F}=\frac{\mu_{F}+\gamma \mu_{G} \circ F}{1+\bar{\mu}_{F} \gamma \mu_{G} \circ F}, \quad \text { where } \quad \gamma=\frac{\bar{F}_{\bar{z}}}{F_{z}} .
$$

Thus

$$
\left\|\mu_{G \circ F}\right\|_{\infty} \leq\left(\left\|\mu_{F}\right\|_{\infty}+\left\|\mu_{G} \circ F\right\|_{\infty}\right)\left(1-\left\|\mu_{F}\right\|_{\infty}\left\|\mu_{G} \circ F\right\|_{\infty}\right)^{-1} .
$$


Let $\omega(t)=\omega_{f, 0}(t)$. Suppose $C>0$ is the constant in the control condition (11). Suppose, in the beginning of the proof, we pick $\delta$ small such that $\omega(C \delta)<1$. From Lemma 2

$$
K_{0}=\tilde{\omega}(\delta)=\sum_{n=0}^{\infty} \omega\left(C \sigma^{n} \delta\right)<\infty
$$

is a convergent series. Thus the product

$$
K_{1}=\prod_{n=0}^{\infty}\left(1-\omega\left(C \sigma^{n} \delta\right)\right)^{-1}<\infty
$$

is also convergent.

Let $\mu(z)=\mu_{\phi_{r}}(z)$ for $z \in \Delta_{\delta}$. Remember that $r=\sigma^{k} \delta$. For $z \in A_{r, 0},|\mu(z)| \leq c_{r}$. For $z \in A_{r,-j}, 1 \leq j \leq k, \phi_{r}(z)=f^{-j}\left(\phi_{r}\left(\lambda^{j} z\right)\right)$. Let $g_{i}(z)=f^{-j+i}\left(\phi_{r}\left(\lambda^{j} z\right)\right)$ for $0 \leq i \leq j$. Let $w=\lambda^{j} z$. Then $\sigma r \leq|w| \leq r$. This implies that $\left|\phi_{r}(w)\right| \leq r$ and

$$
\left|g_{i}(z)\right| \leq C \sigma^{-j+i} r=C \sigma^{k-j+i} \delta
$$

for all $0 \leq i \leq j$. Note that

$$
\left|\mu_{f^{-1}}\right|=\left|\mu_{f} \circ f^{-1}\right| .
$$

By the compsoition formula (2),

$$
\begin{gathered}
|\mu(z)|=\left|\mu_{g_{0}}(z)\right| \leq\left(\left|\mu_{g_{1}}(z)\right|+\left|\mu_{f^{-1}}\left(g_{1}(z)\right)\right|\right)\left(1-\left|\mu_{g_{1}}(z)\right|\left|\mu_{f^{-1}}\left(g_{1}(z)\right)\right|\right)^{-1} \\
=\left(\left|\mu_{g_{1}}(z)\right|+\left|\mu_{f}\left(g_{0}(z)\right)\right|\right)\left(1-\left|\mu_{g_{1}}(z)\right|\left|\mu_{f}\left(g_{0}(z)\right)\right|\right)^{-1} \\
\leq\left|\mu_{g_{1}}(z)\right|\left(1-\omega\left(C \sigma^{k-j} \delta\right)\right)^{-1}+\omega\left(C \sigma^{k-j} \delta\right)\left(1-\omega\left(C \sigma^{k-j} \delta\right)\right)^{-1} .
\end{gathered}
$$

Inductively, we get

$$
\left|\mu_{g_{i}}(z)\right| \leq\left|\mu_{g_{i+1}}(z)\right|\left(1-\omega\left(C \sigma^{k-j+i} \delta\right)\right)^{-1}+\omega\left(C \sigma^{k-j+i} \delta\right)\left(1-\omega\left(C \sigma^{k-j+i} \delta\right)\right)^{-1}
$$

for $0 \leq i \leq j$. So

$$
\begin{aligned}
|\mu(z)| \leq & c_{r} \prod_{l=1}^{j}\left(1-\omega\left(C \sigma^{k-l} \delta\right)\right)^{-1}+\sum_{i=1}^{j} \omega\left(C \sigma^{k-i} \delta\right) \prod_{l=k-j}^{k-i}\left(1-\omega\left(C \sigma^{l} \delta\right)\right)^{-1} \\
& \leq K_{1} c_{r}+K_{1} \sum_{i=1}^{j} \omega\left(C \sigma^{k-i} \delta\right) \leq K_{1}\left(c_{r}+\tilde{\omega}(\delta)\right) \leq K_{1}\left(1+K_{0}\right) .
\end{aligned}
$$

For $z \in A_{r, j}, 1 \leq j<\infty, \phi_{r}(z)=f^{j}\left(\phi_{r}\left(\lambda^{-j} z\right)\right)$. Let $h_{i}(z)=f^{j-i}\left(\phi_{r}\left(\lambda^{-j} z\right)\right)$ for $0 \leq i \leq j$. Let $w=\lambda^{-j} z$. Then $\sigma r \leq|w| \leq r$. This implies that $\left|\phi_{r}(w)\right| \leq r$ and

$$
\left|h_{i}(z)\right| \leq C \sigma^{j-i} r=C \sigma^{k+j-i} \delta
$$

for all $0 \leq i \leq j$. By the compsoition formula (2),

$$
\begin{aligned}
& |\mu(z)|=\left|\mu_{h_{0}}(z)\right| \leq\left(\left|\mu_{h_{1}}(z)\right|+\left|\mu_{f}\left(h_{1}(z)\right)\right|\right)\left(1-\left|\mu_{h_{1}}\right|\left|\mu_{f}\left(h_{1}(z)\right)\right|\right)^{-1} \\
\leq & \left|\mu_{h_{1}}(z)\right|\left(1-\omega\left(C \sigma^{k+j-1} \delta\right)\right)^{-1}+\omega\left(C \sigma^{k+j-1} \delta\right)\left(1-\omega\left(C \sigma^{k+j-1} \delta\right)\right)^{-1} .
\end{aligned}
$$

Inductively, we get

$\left|\mu_{h_{i}}(z)\right| \leq\left|\mu_{h_{i+1}}(z)\right|\left(1-\omega\left(C \sigma^{k+j-i-1} \delta\right)\right)^{-1}+\omega\left(C \sigma^{k+j-i-1} \delta\right)\left(1-\omega\left(C \sigma^{k+j-i-1} \delta\right)\right)^{-1}$. 
So

$$
\begin{aligned}
& |\mu(z)| \leq c_{r} \prod_{l=1}^{j}\left(1-\omega\left(C \sigma^{k+j-l} \delta\right)\right)^{-1}+\sum_{i=1}^{j} \omega\left(C \sigma^{k+j-i} \delta\right) \prod_{l=1}^{i}\left(1-\omega\left(C \sigma^{k+j-l} \delta\right)\right)^{-1} \\
& \leq K_{1} c_{r}+K_{1} \sum_{i=1}^{j} \omega\left(C \sigma^{k+j-i} \delta\right) \leq K_{1}\left(c_{r}+\tilde{\omega}\left(\sigma^{k} \delta\right)\right)=K_{1}\left(c_{r}+\tilde{\omega}(r)\right) \leq K_{1}\left(1+K_{0}\right) .
\end{aligned}
$$

Let $k=K_{1}\left(1+K_{0}\right)$ and $K=(1+k) /(1-k)$. Then $\left\{\phi_{r_{k}}\right\}_{k=1}^{\infty}$ is uniformly $K$ quasiconformal. Consider $B_{r}=\Delta_{\delta} \backslash \Delta_{r}=\cup_{j=-k}^{-1} A_{r, j}$ and $\phi_{r}\left(B_{r}\right)=\cup_{j=-k}^{-1} \phi_{r}\left(A_{r, j}\right)$ for any $r=r_{k}$. Both of the annulli have the same inner circle $S_{r}$. Thus the ratio of the modulus of $\phi_{r}\left(B_{r}\right)$ and the modulus of $B_{r}$ is controlled by two constants from below and above (independent of $r$ but only depends on $K$ ). Therefore, the range of $\phi_{r}$ on $\Delta_{\delta}$ is a Jordan domain bounded above from $\infty$ and below from 0 uniformly in $0<r=r_{k} \leq \delta$. Since, additionally, 0 is fixed by any element in this sequence, the family $\left\{\phi_{r_{k}}\right\}_{k=1}^{\infty}$ is in a compact set in the space of all $K$ quasiconformal homeomorphisms on $\Delta_{\delta}$ (see [1]). Let $\phi$ be a limit mapping of this family. Then we have

$$
f(\phi(z))=\phi(\lambda z), \quad z \in \Delta_{\delta} .
$$

Similar to the arguments above, the complex dilatation of $\phi_{r}(z)$ on disk $\Delta_{\tilde{r}}$ is controlled by $K_{1}\left(c_{\tilde{r}}+\tilde{\omega}(\tilde{r})\right)$ for any $r=r_{k} \leq \tilde{r}$. So the complex dilatation of $\phi$ on $\Delta_{\tilde{r}}$ is also controlled by $K_{1}\left(c_{\tilde{r}}+\tilde{\omega}(\tilde{r})\right) \rightarrow 0$ as $\tilde{r} \rightarrow 0$. Thus $\phi(z)$ is asymptotically conformal at 0 and the proof of existence is completed.

Suppose $\phi_{1}$ and $\phi_{2}$ are two asymptotically conformal conjugacies such that

$$
\phi_{1}^{-1} \circ f \circ \phi_{1}(z)=\lambda z \quad \text { and } \quad \phi_{2}^{-1} \circ f \circ \phi_{2}(z)=\lambda z, \quad z \in \Delta_{\delta} .
$$

Then for $\Phi=\phi_{2}^{-1} \circ \phi_{1}$, we have $\Phi(\lambda z)=\lambda \Phi(z)$. This implies that the complex dilatation $\mu_{\Phi}(z)=\mu_{\Phi}(\lambda z)$, a.e.. This in turn implies that $\mu=0$ a.e. in $\Delta_{\delta}$ and thus $\Phi$ is conformal. Furthermore, $\Phi(z)=a z$ for some $a \neq 0$. This is the uniqueness.

\section{NORMAL FORMS FOR INTEGRABLE ASYMPTOTICALLY CONFORMAL SUPER-ATTRACTING FIXED POINTS}

The other main result in this article is Theorem 2, which says that if $g=f\left(z^{n}\right)$ is a quasiregular map and 0 is an integrable asymptotically conformal super-attracting fixed point, then $g$ can be written into the normal form $z: \rightarrow z^{n}$ under some quasiconformal change of coordinate which is asymptotically conformal at 0 . The result generalizes the famous Böttcher's Theorem in classical analysis. Again, to present a clear idea about how we get Theorem 2, we first use the same idea to give another proof of Böttcher's Theorem, which is first given in [20. The idea of the new proof follows the viewpoint of holomorphic motions. For the classical proof of Böttcher's Theorem, the reader may refer to Böttcher's original paper [8] or most recent books [9, 34. Actually from the technical point of views, our proof is more complicate and uses a sophistical result. But from the conceptual point of views, our proof gives some inside mechanism of the normal form for a superattracting fixed point. The idea of the proof is basically the same as that in the previous section, but the actual proof is little bit different. The reason is that in the previous case, $f$ is a homeomorphism so we can iterate both forward and backward, but in Theorem 2 or Böttcher's Theorem, $g$ is not a homeomorphism. 
Theorem 5 (Böttcher's Theorem). Suppose $g(z)=\sum_{j=n}^{\infty} a_{j} z^{j}, a_{n} \neq 0, n \geq 2$, is analytic on a disk $\Delta_{\delta_{0}}, \delta_{0}>0$. Then there exists a conformal map $\phi: \Delta_{\delta} \rightarrow \phi\left(\Delta_{\delta}\right)$ for some $\delta>0$ such that

$$
\phi^{-1} \circ g \circ \phi(z)=z^{n}, \quad z \in \Delta_{\delta}
$$

The conjugacy $\phi^{-1}$ is unique up to multiplication by $(n-1)^{\text {th }}$-roots of the unit.

A new proof of Böttcher's Theorem. Conjugating by $z \rightarrow b z$, we can assume $a_{n}=$ 1, i.e.,

$$
g(z)=z^{n}+\sum_{j=n+1}^{\infty} a_{j} z^{j}
$$

We use $\Delta_{r}^{*}=\Delta_{r} \backslash\{0\}$ to mean a punctured disk of radius $r>0$. Write

$$
g(z)=z^{n}\left(1+\sum_{j=1}^{\infty} a_{j+n} z^{j}\right)
$$

Assume $0<\delta_{1}<\min \left\{1 / 2, \delta_{0} / 2\right\}$ is small enough such that

$$
1+\sum_{j=1}^{\infty} a_{j+n} z^{j} \neq 0 \quad \text { and } \quad \frac{1}{\sqrt[n]{\left|1+\sum_{j=1}^{\infty} a_{j+n} z^{j}\right|}} \geq \frac{1}{2}, \quad z \in \Delta_{2 \delta_{1}} .
$$

Then $g: \Delta_{2 \delta_{1}}^{*} \rightarrow g\left(\Delta_{2 \delta_{1}}^{*}\right)$ is a covering map of degree $n$.

Let $0<\delta<\delta_{1}$ be a fixed number such that $g^{-1}\left(\Delta_{\delta}\right) \subset \Delta_{\delta_{1}}$. Since

$$
z \rightarrow z^{n}: \Delta_{\sqrt[n]{\delta}}^{*} \rightarrow \Delta_{\delta}^{*} \quad \text { and } \quad g: g^{-1}\left(\Delta_{\delta}^{*}\right) \rightarrow \Delta_{\delta}^{*}
$$

are both of covering maps of degree $n$, the identity map of $\Delta_{\delta}$ can be lifted to a holomorphic diffeomorphism

$$
h: \Delta_{\sqrt[n]{\delta}}^{*} \rightarrow g^{-1}\left(\Delta_{\delta}^{*}\right)
$$

i.e., $h$ is a map such that the diagram

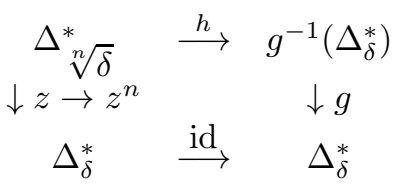

commutes. We pick the lift so that

$$
h(z)=z\left(1+\sum_{j=2}^{\infty} b_{j} z^{j-1}\right)=z \psi(z) .
$$

From

$$
g(h(z))=z^{n}, \quad z \in \Delta_{\sqrt[n]{\delta}}^{*}
$$

we get

$$
|h(z)|=\frac{|z|}{\sqrt[n]{\left|1+\sum_{j=1} a_{n+j}(h(z))^{j}\right|}} \geq \frac{|z|}{2} .
$$

For any

$$
0<r \leq \min \left\{\left(\frac{1}{2}\right)^{\frac{n}{(n-1)}}, \delta^{n}\right\}
$$


let $S_{r}=\{z \in \mathbb{C}|| z \mid=r\}$ and $T_{r}=\{z \in \mathbb{C}|| z \mid=\sqrt[n]{r}\}$. Consider the set $E=S_{r} \cup T_{r}$ and the map

$$
\phi_{r}(z)= \begin{cases}z, & z \in S_{r} \\ z \psi(z), & z \in T_{r}\end{cases}
$$

Define

$$
h_{r}(c, z)=\left\{\begin{array}{ll}
z, & z \in S_{r} \\
z \psi\left(\frac{c z}{\sqrt[n]{r}}\right), & z \in T_{r}
\end{array}: \Delta \times E \rightarrow \hat{\mathbb{C}} .\right.
$$

Note that

$$
z \psi\left(\frac{c z}{\sqrt[n]{r}}\right)=\frac{\sqrt[n]{r}}{c} h\left(\frac{c z}{\sqrt[n]{r}}\right), \quad z \in T_{r}, c \neq 0
$$

This implies that

$$
\left|h_{r}(c, z)\right|=\frac{\sqrt[n]{r}}{|c|} h\left(\frac{c z}{\sqrt[n]{r}}\right) \mid \geq \frac{\sqrt[n]{r}}{|c|} \frac{|c z|}{2 \sqrt[n]{r}} \geq \frac{\sqrt[n]{r}}{2}>r, \quad z \in T_{r} .
$$

So images of $S_{r}$ and $T_{r}$ under $h_{r}(c, z)$ do not cross each other.

Now let us check $h_{r}(c, z)$ is a holomorphic motion. First $h_{r}(0, z)=z$ for $z \in E$. For fixed $x \in E, h_{r}(c, z)$ is holomorphic on $c \in \Delta$. For fixed $c \in \Delta, h_{r}(c, z)$ restricted to $S_{r}$ and $T_{r}$, respectively, are injective. But the images of $S_{r}$ and $T_{r}$ under $h_{r}(c, z)$ do not cross each other. So $h_{r}(c, z)$ is injective on $E$. Thus

$$
h_{r}(c, z): \Delta \times E \rightarrow \hat{\mathbb{C}}
$$

is a holomorphic motion. By Theorem 3 it can be extended to a holomorphic motion

$$
H_{r}(c, z): \Delta \times \hat{\mathbb{C}} \rightarrow \hat{\mathbb{C}} .
$$

And moreover, for each $c \in \Delta, H_{r}(c, \cdot)$ is a quasiconformal map whose quasiconformal dilatation satisfies

$$
K\left(H_{r}(c, \cdot)\right) \leq \frac{1+|c|}{1-|c|} .
$$

Now consider $H_{r}(\sqrt[n]{r}, \cdot)$. It is a quasiconformal map with quasiconformal constant

$$
K_{r} \leq \frac{1+\sqrt[n]{r}}{1-\sqrt[n]{r}}
$$

Let

$$
A_{r, j}=\left\{z \in \mathbb{C}\left|\sqrt[n^{j}]{r} \leq\right| z \mid \leq \sqrt[n^{j+1}]{r}\right\}, \quad j=0,1,2, \cdots .
$$

Consider the restriction $\phi_{r, 0}=H_{r}(\sqrt[n]{r}, \cdot) \mid A_{r, 0}$. It is an extension of $\phi_{r}$, i.e., $\phi_{r, 0} \mid E=$ $\phi_{r}$.

Let $\tilde{A}_{r, 0}$ be the annulus bounded by $S_{r}$ and $g^{-1}\left(S_{r}\right)$ and define $\tilde{A}_{r, j}=g^{-j}\left(\tilde{A}_{r, 0}\right)$, $j \geq 0$. Since $z \rightarrow z^{n}: A_{r, 1} \rightarrow A_{r, 0}$ and $g: \tilde{A}_{r, 1} \rightarrow \tilde{A}_{r, 0}$ are both covering maps of degree $n$, so $\phi_{r, 0}$ can be lifted to a quasiconformal map $\phi_{r, 1}: A_{r, 1} \rightarrow \tilde{A}_{r, 1}$, i.e., the following diagram

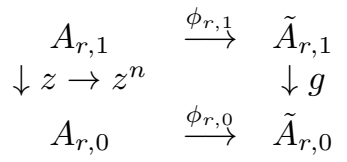

commutes. We pick the lift $\phi_{r, 1}$ such that it agrees with $\phi_{r, 0}$ on $T_{r}$. The quasiconformal dilatation of $\phi_{r, 1}$ is less than or equal to $K_{r}$. 
For an integer $k>0$, take $r=r_{k}=\delta^{n^{k}}$. Inductively, we can define a sequence of $K_{r}$-quasiconformal maps $\left\{\phi_{r, j}\right\}_{j=0}^{k}$ such that

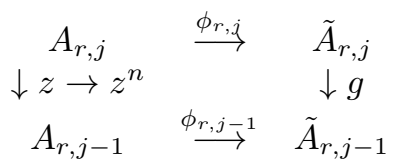

commutes and $\phi_{r, j}$ and $\phi_{r, j-1}$ agree on the common boundary of $A_{r, j}$ and $A_{r, j-1}$. Note that

$$
\bar{\Delta}_{\delta}=\Delta_{r} \cup \cup_{j=0}^{k} A_{r, j}
$$

Now we can define a quasiconformal map, which we still denote by $\phi_{r}$ as follows.

$$
\phi_{r}(z)= \begin{cases}z, & z \in \Delta_{r} ; \\ \phi_{r, j}, & z \in A_{r, j}, \quad j=0,1, \cdots, k .\end{cases}
$$

The quasiconformal dilatation of $\phi_{r}$ on $\Delta_{\delta}$ is less than or equal to $K_{r}$ and

$$
g\left(\phi_{r}(z)\right)=\phi_{r}\left(z^{n}\right), \quad z \in \cup_{j=1}^{k} A_{r, j} .
$$

Since $g(z)=z^{n}(1+O(z)), g^{k}(z)=z^{n^{k}} \prod_{i=0}^{k-1}\left(1+O\left(z^{n^{i}}\right)\right)$. Because $\sqrt[n^{k}]{r_{k}}=\delta$, the range of $\phi_{r_{k}}$ on $\Delta_{\delta}$ is a Jordan domain bounded above from $\infty$ and below from 0 uniformly in $k$. In addition, 0 is fixed by $\phi_{r_{k}}$ and the quasiconformal dilatations of the $\phi_{r_{k}}$ are uniformly bounded in $k$. Therefore, the sequence $\left\{\phi_{r_{k}}\right\}_{k=1}^{\infty}$ is in a compact set in the space of all quasiconformal homeomorphisms on $\Delta_{\delta}$ (see [1]). Let $\phi$ be a limiting map of this family. Then we have

$$
g(\phi(z))=\phi\left(z^{n}\right), \quad z \in \Delta_{\delta} .
$$

Since the quasiconformal dilatation of $\phi$ is less than or equal to $\left(1+\sqrt[n]{r_{k}}\right) /\left(1-\sqrt[n]{r_{k}}\right)$ for all $k>0$, it follows that $\phi$ is a 1-quasiconformal map, and thus conformal. This is the proof of the existence.

Suppose $\phi_{1}$ and $\phi_{2}$ are two conjugacies such that

$$
\phi_{1}^{-1} \circ g \circ \phi_{1}(z)=z^{n} \quad \text { and } \quad \phi_{2}^{-1} \circ g \circ \phi_{2}(z)=z^{n}, \quad z \in \Delta_{\delta} .
$$

For

$$
\Phi(z)=\phi_{2}^{-1} \circ \phi_{1}(z)=\sum_{j=1}^{\infty} a_{j} z^{j}
$$

we have $\Phi\left(z^{n}\right)=(\Phi(z))^{n}$. This implies $a_{1}^{n}=a_{1}$ and $a_{j}=0$ for $j \geq 2$. Since $a_{1} \neq 0$, we have $a_{1}^{n-1}=1$ and $\phi_{2}^{-1}=a_{1} \phi_{1}^{-1}$. This is the uniqueness.

We now prove Theorem 2. The proof follows almost the same footsteps of those of Theorem 1 and Theorem 5 .

Proof of Theorem 2, Let $g=f \circ q_{n}, n \geq 2$. Conjugating by $z \rightarrow b z$, we can assume $f^{\prime}(0)=\lim _{|z| \rightarrow 0} f(z) / z=1$.

We use $\Delta_{r}^{*}=\Delta_{r} \backslash\{0\}$ to mean a punctured disk of radius $r>0$. There is a $0<\delta_{1}<1$ such that $g: \Delta_{2 \delta_{1}}^{*} \rightarrow g\left(\Delta_{2 \delta_{1}}^{*}\right)$ is a covering map of degree $n$.

Let $0<\delta<\delta_{1}$ be a fixed number such that $g^{-1}\left(\Delta_{\delta}\right) \subset \Delta_{\delta_{1}}$. Since

$$
z \rightarrow z^{n}: \Delta_{\sqrt[n]{\delta}}^{*} \rightarrow \Delta_{\delta}^{*} \quad \text { and } \quad g: g^{-1}\left(\Delta_{\delta}^{*}\right) \rightarrow \Delta_{\delta}^{*}
$$


are both of covering maps of degree $n$, the identity map of $\Delta_{\delta}$ can be lifted to a homeomorphism

$$
h: \Delta_{\sqrt[n]{\delta}}^{*} \rightarrow g^{-1}\left(\Delta_{\delta}^{*}\right)
$$

Furthermore, $h$ is a quasiconformal map and integrable asymptotically conformal at 0 such that the diagram

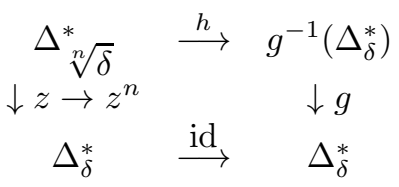

commutes. We pick the lift so that

$$
h^{\prime}(0)=\lim _{z \rightarrow 0} \frac{h(z)}{z}=1 .
$$

These can be seen from the equation

$$
g(h(z))=z^{n}, \quad z \in \Delta_{\sqrt[n]{\delta}}^{*}
$$

For any $0<r \leq \delta$, let $S_{r}=\{z \in \mathbb{C}|| z \mid=r\}$ and $T_{r}=\{z \in \mathbb{C}|| z \mid=\sqrt[n]{r}\}$. Consider the set $E=S_{r} \cup T_{r}$ and the map

$$
\phi_{r}(z)= \begin{cases}z, & z \in S_{r} \\ h(z), & z \in T_{r} .\end{cases}
$$

It is clear that

$$
g\left(\phi_{r}(z)\right)=\phi_{r}\left(z^{n}\right)
$$

for $z \in T_{r}$.

Extend $h$ to $\hat{\mathbb{C}}$ by quasiconformal reflection with respect to $S_{r}$ and $\phi\left(S_{r}\right)$ (see [1]). We still denote this extended map as $\phi$. Let $\nu=\phi_{\bar{z}} / \phi_{z}$ be the complex dilatation of the extended $\phi$. Then

$$
a(r)=\|\nu\|_{\infty}=O\left(\|\mu \mid \Delta \sqrt[n]{r}\|_{\infty}\right)=O(\omega(\sqrt[n]{r})) .
$$

Assume $h(\sqrt[n]{r})=\tau_{r}$. Consider $\nu_{c}=c a_{0} a(r)^{-1} \nu$ and the unique solution $\phi_{c}=$ $\phi^{\nu_{c}}$ that maps $0, r$, and $\infty$ to $0, \tau_{r}$, and $\infty$, respectively. Here $a_{0}$ is a constant independent of $r$ such that $\left|\phi_{c}(z)\right|>r$ for all $|z|=\sqrt[n]{r}$ and $|c|<1$. (Since $\phi_{c}$ can be written as a power series in $\nu_{c}$ and $\left\|\nu_{c}\right\| \rightarrow 0$ uniformly as $r \rightarrow 0$, such an $a_{0}$ exists.) Then $\phi_{c}$ holomorphically depends on $c \in \Delta$. Define

$$
\phi_{r}(c, z)= \begin{cases}z & z \in S_{r} \\ \phi_{c}(z), & z \in T_{r}\end{cases}
$$

It is a holomorphic motion from $\Delta \times E \rightarrow \hat{\mathbb{C}}$. From Theorem 3, $\phi(c, z)$ can be extended to a holomorphic motion from $\Delta \times \hat{\mathbb{C}} \rightarrow \hat{\mathbb{C}}$, which we still denote by $\phi(c, z)$, such that the quasiconformal dilatation of $\phi(c, \cdot)$ is less than or equal to $(1+|c|) /(1-|c|)$. In particular when $c_{r}=a_{0}^{-1} a(r), \phi_{r}\left(c_{r}, z\right) \mid E=\phi_{r}$. We still use $\phi_{r}$ to denote $\phi_{r}\left(c_{r}, \cdot\right) \mid A_{r, 0}$.

For an integer $k>0$, take $r=r_{k}=\delta^{n^{k}}$. Let

$$
A_{r, j}=\left\{z \in \mathbb{C}\left|\sqrt[n^{j}]{r} \leq\right| z \mid \leq \sqrt[n^{j+1}]{r}\right\}
$$

for $0 \leq j \leq k-1$. Then

$$
\Delta_{\delta}=\Delta_{r} \cup \cup_{j=0}^{k-1} A_{r, j}
$$


Let $\phi_{r}(z)=z$ for $z \in \Delta_{r}$ and extend $\phi_{r}$ to $\cup_{j=0}^{k-1} A_{r, j}$ by lifting. Then we get a homeomorphism on $\Delta_{\delta}$, which we still denote as $\phi_{r}$ (refer to the proof of Theorem 11). Formally we can use the following formula to define $\phi_{r}$,

$$
\phi_{r}(z)=g^{-j}\left(\phi_{r}\left(z^{n^{j}}\right)\right), \quad z \in A_{r, j}, \quad j=0, \cdots, k-1,
$$

and $\phi_{r}(z)=z$ for $z \in \Delta_{r}$. Since $\phi_{r} \mid E$ is a conjugacy from $g$ to $q_{n}(z)=z^{n}, \phi_{r}$ is continuous on $\Delta_{\delta}$.

Let $\omega(t)=\omega_{f, 0}(t)$ for $0<t \leq \delta$. Suppose $C>0$ is a constant such that

$$
C^{-1} \leq\left|\frac{f(z)}{z}\right| \leq C
$$

for $z \in \bar{\Delta}_{\delta}$. Suppose, in the beginning of the proof, we pick $\delta$ small such that $\omega(C \delta)<1$. Let $0<\delta<\sigma<1$ be a fixed constant. From Lemma 2 ,

$$
K_{0}=\tilde{\omega}(\delta)=\sum_{n=0}^{\infty} \omega\left(C \sigma^{n} \delta\right)<\infty
$$

is a convergent series. Thus the product

$$
K_{1}=\prod_{n=0}^{\infty}\left(1-\omega\left(C \sigma^{n} \delta\right)\right)^{-1}<\infty
$$

is also convergent.

Using the similar argument to that in the proof of Theorem 1, we can obtain that the complex dilatation $\mu(z)=\mu_{\phi_{r}}(z)$ over $\Delta_{\delta}$ can be controlled by

$$
|\mu(z)| \leq K_{1}\left(c_{r}+\tilde{\omega}(\delta)\right) \leq K_{1}\left(1+K_{0}\right)
$$

for $z \in \Delta_{\delta}$ and

$$
|\mu(z)| \leq K_{1}\left(c_{\tilde{r}}+\tilde{\omega}(\tilde{r})\right)
$$

for $z \in \Delta_{\tilde{r}}$ and all $r=r_{k} \leq \tilde{r}$.

Let $k=K_{1}\left(1+K_{0}\right)$ and $K=(1+k) /(1-k)$. Then $\left\{\phi_{r_{k}}\right\}_{k=1}^{\infty}$ is uniformly $K$-quasiconformal. Consider $B_{r}=\Delta_{\delta} \backslash \Delta_{r}=\cup_{j=0}^{k-1} A_{r, j}$ and $\phi_{r}\left(B_{r}\right)=\cup_{j=0}^{k-1} \phi_{r}\left(A_{r, j}\right)$ for any $r=r_{k}$. Both of the annulli have the same inner circle $S_{r}$. Thus the ratio of the modulus of $\phi_{r}\left(B_{r}\right)$ and the modulus of $B_{r}$ is controlled by two constants from below and above (independent of $r$ but only depends on $K$ ). Therefore, the range of $\phi_{r}$ on $\Delta_{\delta}$ is a Jordan domain bounded above from $\infty$ and below from 0 uniformly in $0<r=r_{k} \leq \delta$. Since, additionally, 0 is fixed by any element in this sequence, the sequence $\left\{\phi_{r_{k}}\right\}_{k=1}^{\infty}$ is in a compact set in the space of all $K$-quasiconformal homeomorphisms on $\Delta_{\delta}$ (see [1]). Let $\phi$ be a limit mapping of this family. Then we have

$$
g(\phi(z))=\phi\left(z^{n}\right), \quad z \in \Delta_{\delta}
$$

Similar to the arguments in Theorem 1, the complex dilatation of $\phi_{r}(z)$ on disk $\Delta_{\tilde{r}}$ is controlled by $K_{1}\left(c_{\tilde{r}}+\tilde{\omega}(\tilde{r})\right)$ for any $r=r_{k} \leq \tilde{r}$. So the complex dilatation of $\phi$ on $\Delta_{\tilde{r}}$ is also controlled by $K_{1}\left(c_{\tilde{r}}+\tilde{\omega}(\tilde{r})\right) \rightarrow 0$ as $\tilde{r} \rightarrow 0$. Thus $\phi(z)$ is asymptotically conformal at 0 . The proof of existence is completed.

Suppose $\phi_{1}$ and $\phi_{2}$ are two asymptotically conformal conjugacies such that

$$
\phi_{1}^{-1} \circ g \circ \phi_{1}(z)=z^{n} \quad \text { and } \quad \phi_{2}^{-1} \circ g \circ \phi_{2}(z)=z^{n}, \quad z \in \Delta_{\delta} .
$$

Then for $\Phi=\phi_{2}^{-1} \circ \phi_{1}$, we have $\Phi\left(z^{n}\right)=(\Phi(z))^{n}$. This implies that the complex dilatation $\left\|\mu_{\Phi}(z)\right\|=\left\|\mu_{\Phi}\left(z^{n}\right)\right\|$, a. e.. This in turn implies that $\mu=0$ a.e. in $\Delta_{\delta}$ 
and thus $\Phi$ is conformal, and therefore, $\Phi(z)=a z$ with $a^{n-1}=1$. This is the uniqueness.

\section{REFERENCES}

[1] L. V. Ahlfors, Lectures on Quasiconformal Mappings, Van Nostrand Mathematical studies, 10, D. Van Nostrand Co. Inc., Toronto-New York-London, 1966.

[2] L. V. Ahlfors and L. Bers, Riemann's mapping theorem for variable metrics, Annals of Math. (2), 72 (1960), 385-404.

[3] K. Astala, Planar quasiconformal mappings; deformations and interactions, In Quasiconformal Mappings and Analysis-A collection of papers honoring F. W. Gehring, 1998, SpringerVerlag, New York, Inc., 33-54.

[4] K. Astala and G. J. Martin, Holomorphic motions, Papers on Analysis, 27-40, Rep. Univ. Jyväsklä Dep. Math. Stat., 83, Univ. Jyväskylä, 2001.

[5] L. Bers, On a theorem of Mori and the definition of quasiconformality, Trans. Amer. Math. Soc., 84 (1957), 78-84.

[6] L. Bers and H. L. Royden, Holomorphic families of injections, Acta Math, 157 (1986), 259286.

[7] V. B. Bojarski, Generalized solutions of a system of differential equations of the first order and elliptic type with discontinuous coefficients, Math. Sbornik, 85 (1957), 451-503.

[8] L. E. Böttcher, The principal laws of convergence of iterates and their aplication to analysis (Russian), Izv. Kazan. Fiz.-Mat. Obshch. 14) (1904), 155-234.

[9] L. Carleson and T. Gamelin, Complex Dynamics, Universitext: Tracts in Mathematics, Springer-Verlag, New York, 1993.

[10] E. M. Chirka, On the extension of holomorphic motions, Doklady Akademii Nauk, 397(1):3740, 2004.

[11] S. K. Donaldson and D. P. Sullivan, Quasiconformal 4-manifolds, Acta Math. 163 (1989), 181-252.

[12] A. Douady, Prolongement de mouvements holomorphes [d'après Slodkowski et autres], Séminarie N. Bourbaki (1993/1994), Astérique No. 227 (1995) \& Exp. No. 755 3, 7-20.

[13] P Duren, J. Heinonen, B. Osgood, and B. Palka, Quasiconformal Mappings and Analysis, Springer, 1997

[14] C. J. Earle, I. Kra, and S. L. Krushkal, Holomorphic motions and Teichmüller spaces, Trans. Amer. Math. Soc., 343 (2) (1994), 927-948.

[15] C. J. Earle and S. Mitra, Variation of moduli under holomorphic motions, Comtemp. Math., 256 (2000), 39-67.

[16] F. Gardiner, Y. Jiang, and Z. Wang, Holomorphic motions and related topics, arXiv:0802.2111v1, Preprint.

[17] F. Gardiner and L. Keen, Holomorphic motions, Lipman Bers, Selected Works Part I and II, Edited by I. Kra and B. Maskit, AMS, 1999.

[18] F. Gardiner and N. Lakic, Quasiconformal Teichmüller Theory, Mathematicak Survey and Monographs, American Mathematical Society, Rhode Island, 76 (2000).

[19] V. Ya. Gulyanskii, O. Martio, V. I. Ryazanov, and M. Vuorinen, On the asymptotic behavior of quasiconformal mappings in space in Quasiconformal Mappings and Analysis (edited by $\mathrm{P}$ Duren, J. Heinonen, B. Osgood, and B. Palka), Springer, 1997, 159-180.

[20] Y. Jiang, Holomorphic motions and normal forms in complex analysis, Studies in Advanced Mathematics, ICCM20042008, AMS/IP, Vol. 42 (2008), Part 2, 457-466.

[21] Y. Jiang, Holomorphic motions, Fatou linearization, and quasiconformal rigidity for parabolic germs, arXiv:0707.4349v 4, Preprint.

[22] G. Königs, Recherches sur les integrals de certains equations fonctionelles, Ann. Sci. Éc. Norm. Sup., ( $3^{e}$ ser.) 1 (1884) supplém. 1-41.

[23] O. Lehto, Univalent Functions and Teichmüller Spaces, Springer-Verlag, New York, Berlin, 1987.

[24] C. McMullen and D. Sullivan, Quasiconformal homeomorphisms and dynamics III: The Teichmüller space of a holomorphic dynamical systems, Adv. Math., 135 (1998), 351-395.

[25] Ch. Pommerenke, Boundary Behaviour of Conformal Maps, Springer-Verlag, 1992.

[26] Yu. G. Reshetnyak, Stability theorems in geometry and analysis, Nauka, Sibirskoe otdelenie, Novosibirsk, Russian, 1982. 
[27] M. A. Lavrent'ev, Sur une classe de représentation continues, Mat. Sbnornik 42(1935), pp. 407-423.

[28] M. A. Lavrent'ev, A fundamental theorem of the theory of quasi-conformal mapping of plane regions. Izvestya Akademii Nauk S.S.S.R. 12 (1948), pp. 513-554. (Russian).

[29] Z. Li, Quasiconformal Mappings and Applications in Riemann Surfaces, Science publciation of China, 1988.

[30] G. Lieb, Holomorphic motions and Teichmüller space, Ph.D. dissertation, Cornell University, 1990.

[31] R. Mañe, P. Sad, and D. Sullivan, On the dynamics of rational maps, Ann. Éc. Norm. Sup., 96 (1983), 193-217.

[32] C. McMullen, Renormalization and 3-Manifolds which Fiber over the Circle, Annals of Mathematics Studies, Number 143, Princeton University, Priceton, New Jersey.

[33] W. de Melo and S. van Strien One-Dimensional Dynamics, Springer-Verlag, Berlin, New York, 1993.

[34] J. Milnor, Dynamics in One Complex Variable, Introductory Lectures, Vieweg, 2nd Edition, 2000.

[35] S. Mitra, Techmüller spaces and holomorphic motions, Journal d'Analyse Mathématique, 81 (2000), 1-33.

[36] C. B. Morrey, On the solutions of quasi-linear elliptic partial differential equations, Trans. Amer. Math. Math. Soc., 43 (1938), 126-166.

[37] S. Nag, The Complex Analytic Theory of Teichmüller Spaces, John Wiley and Sons, New York, 1988.

[38] Z. Slodkowski, Holomorphic motions and polynomial hulls, Proc. Amer. Math. Soc., 111 (1991), 347-355.

[39] D. Sullivan and W. Thurston, Extending holomorphic motions, Acta Math., 157 (1986), 243-257.

Department of Mathematics, Queens College of the City University of New York, Flushing, NY 11367-1597, and, Department of Mathematics, Graduate School of the City University of New York, 365 Fifth Avenue, New York, NY 10016

E-mail address: Yunping.Jiang@qc.cuny.edu 